PROCEEDINGS OF THE

AMERICAN MATHEMATICAL SOCIETY

Volume 131, Number 5, Pages 1607-1610

S 0002-9939(02)06656-X

Article electronically published on September 19, 2002

\title{
A NOTE ON REGULAR DIRICHLET SUBSPACES
}

\author{
MASATOSHI FUKUSHIMA AND JIANGANG YING
}

(Communicated by Claudia M. Neuhauser)

\begin{abstract}
In this short article we shall consider the Dirichlet space associated with the distorted Brownian motion on a one-dimensional closed interval and prove that it admits no proper regular Dirichlet subspaces.
\end{abstract}

Let $X$ be a locally compact separable metric space and $m$ a fully supported positive Radon measure on $X . C_{0}(X)$ will denote the space of continuous functions on $X$ with compact support. A Markovian symmetric closed form $(\mathcal{E}, \mathcal{F})$ on $L^{2}(X ; m)$ is called a Dirichlet form, while it is called regular if $\mathcal{F} \cap C_{0}(X)$ is dense both in $C_{0}(X)$ with uniform norm and in $\mathcal{F}$ with $\mathcal{E}_{1}$-norm. The regularity guarantees the existence of a unique associated symmetric Hunt process on $X$.

Assume that $(\mathcal{E}, \mathcal{F})$ is a regular Dirichlet form on $L^{2}(X, m)$ and $\hat{\mathcal{F}}$ a linear subspace of $\mathcal{F}$. If $(\mathcal{E}, \hat{\mathcal{F}})$ is also a regular Dirichlet form on $L^{2}(X, m)$, it is natural to ask whether or not $\hat{\mathcal{F}}$ coincides with $\mathcal{F}$. If not, what condition would guarantee the coincidence? The question was originally raised in [3] where it was asked if strong subordination is equivalent to subordination.

In this article, we will prove that the regular Dirichlet form corresponding to distorted Brownian motion (including the ordinary reflecting Brownian motion) on a 1-dimensional closed interval admits no proper regular Dirichlet subspaces. But the problem remains open in the higher dimensional cases.

Let us consider a one dimensional interval $I=(0,1)$ and a function $\rho$ on $I$ such that

$$
\rho \geq 0, \quad \rho, \frac{1}{\rho} \in L^{1}(I)
$$

We let

$$
\mathcal{E}(u, v)=\frac{1}{2} \int_{I} u^{\prime} v^{\prime} \rho d x
$$

for $u, v \in \mathcal{F}$, where

$$
\mathcal{F}=\left\{u \in L^{2}(I ; \rho d x): u \text { is absolutely continuous, } u^{\prime} \in L^{2}(I ; \rho d x)\right\} .
$$

Lemma 1. $(\mathcal{E}, \mathcal{F})$ is a regular Dirichlet space on $L^{2}(\bar{I} ; \rho d x)$.

Received by the editors August 3, 2001 and, in revised form, December 18, 2001.

1991 Mathematics Subject Classification. Primary 60J45, 60J65, 31B15.

Key words and phrases. Regular Dirichlet space, distorted Brownian motion.

The research of the first author was supported in part by Grant-in-Aid for Scientific Research (C) (2).

The research of the second author was supported in part by NNSF of China 19501036.

(C)2002 American Mathematical Society 
Proof. We have

$$
\left|\int_{I} u \phi d x\right| \leq C \sqrt{\int_{I} u^{2} \rho d x}, \quad u \in L^{2}(I ; \rho d x), \quad \phi \in C_{b}(I),
$$

for $C=\sup _{x \in I}|\phi(x)| \sqrt{\int_{I} \frac{1}{\rho} d x}$. Suppose that $u_{n} \in \mathcal{F}, n=1,2, \cdots$, constitute an $\mathcal{E}$-Cauchy sequence and $u_{n} \rightarrow u \in L^{2}(I ; \rho d x)$ in $L^{2}(I ; \rho d x)$. Then $u_{n}^{\prime} \rightarrow v$ for some $v \in L^{2}(\rho d x)$ in $L^{2}(\rho d x)$. By the above inequality

$$
\int_{I} v \phi d x=\lim _{n \rightarrow \infty} \int_{I} u_{n}^{\prime} \phi d x=-\lim _{n \rightarrow \infty} \int_{I} u_{n} \phi^{\prime} d x=-\int_{I} u \phi^{\prime} d x
$$

for each $\phi \in C_{0}^{1}(I)$, and we see that $u \in \mathcal{F}$ and $u^{\prime}=v$.

Notice that $\mathcal{F} \subset C(\bar{I})$ because of the inequality

$$
(u(x)-u(y))^{2}=\left(\int_{x}^{y} u^{\prime}(t) \sqrt{\rho(t)} \frac{1}{\sqrt{\rho(t)}} d t\right)^{2} \leq \mathcal{E}(u, u) \cdot \int_{x}^{y} \frac{1}{\rho(x)} d x,
$$

for $u \in \mathcal{F}, 0 \leq x<y \leq 1$. Since $\mathcal{F}$ is an algebra separating points of $\bar{I}$, it is dense in $C(\bar{I})$.

Theorem 2. Let $\hat{\mathcal{F}}$ be a subspace of $\mathcal{F}$ such that $(\mathcal{E}, \hat{\mathcal{F}})$ is a regular Dirichlet space on $L^{2}(\bar{I}, \rho d x)$. Then $\hat{\mathcal{F}}=\mathcal{F}$.

Proof. First, $(\mathcal{E}, \hat{\mathcal{F}})$ is a regular local Dirichlet space on $L^{2}(\bar{I})$. Second, it is irreducible because $\hat{\mathcal{F}}$ contains no discontinuous function. The inequality (2) implies that each one point of $\bar{I}$ has a positive capacity. Since $\hat{\mathcal{F}}$ is dense in $C(\bar{I})$, there exists $u \in \hat{\mathcal{F}}$ with $u \geq 1$ and hence $1=u \wedge 1 \in \hat{\mathcal{F}}$ and $\mathcal{E}(1,1)=0$. Therefore $(\hat{\mathcal{F}}, \mathcal{E})$ is point recurrent.

Let $\mathbf{M}=\left(X_{t}, P_{x}\right)$ be the associated diffusion process on $\bar{I}$. Let

$$
\hat{\mathcal{F}}_{0}=\{u \in \hat{\mathcal{F}}: u(0)=0\} .
$$

$\left(\hat{\mathcal{F}}_{0}, \mathcal{E}\right)$ is a regular Dirichlet space on $L^{2}((0,1])$ and associated with the part of $\mathbf{M}$ on the set $(0,1]$. By inequality (2) again, we see that the extended Dirichlet space $\hat{\mathcal{F}}_{0, e}$ of $\hat{\mathcal{F}}_{0}$ is transient and equal to $\hat{\mathcal{F}}_{0}$.

We shall consider a hitting probability with respect to $\mathbf{M}$. Let $\sigma_{0}$ and $\sigma_{1}$ be the hitting time of $\{0\}$ and $\{1\}$ respectively, and define

$$
s(x)=P_{x}\left(\sigma_{1}<\sigma_{0}\right) \quad x \in(0,1] .
$$

Then, $s$ coincides with the 0 -order equilibrium potential of $\{1\}$ with respect to $\left(\hat{\mathcal{F}}_{0}, \mathcal{E}\right)$ and satisfies that $s \in \hat{\mathcal{F}}_{0}$ and $\mathcal{E}(s, v)=0$ for all $v \in \hat{\mathcal{F}}$ with $v(0)=v(1)=0$. On the other hand, $s$ is strictly increasing on $I$ and $s(0)=0, s(1)=1$ since any singleton has positive capacity. $\psi(s) \in \hat{\mathcal{F}}_{0}$ for all $\psi \in C^{1}(\mathbb{R})$ with $\psi(0)=0$, because $\psi \circ s$ is a normal contraction of $M \cdot s$ for $M=\sup _{x \in I}\left|\psi^{\prime}(x)\right|$. Therefore

$$
\int_{I} s^{\prime}(x) \psi^{\prime}(s(x)) s^{\prime}(x) \rho(x) d x=0
$$

for all $\psi \in C_{0}^{1}(I)$, namely,

$$
\int_{I} s^{\prime}(t(x)) \rho(t(x)) \psi^{\prime}(x) d x=0
$$


for all $\psi \in C_{0}^{1}(I)$, where $t(x)=s^{-1}(x)$. Hence $s^{\prime}(t(x)) \rho(t(x))$ is a constant on $I$ and so is $s^{\prime}(x) \rho(x)$.

Consequently we obtain

$$
s(x)=c_{0} \int_{0}^{x} \frac{1}{\rho(y)} d y, \quad x \in I, \quad c_{0}=\left(\int_{0}^{1} \frac{1}{\rho(y)} d y\right)^{-1} .
$$

Let

$$
\mathcal{F}_{0}=\{u \in \mathcal{F}: u(0)=0\} .
$$

By inequality (2) again, we see that the metrics $\mathcal{E}_{1}$ and $\mathcal{E}$ are equivalent on the space $\mathcal{F}_{0}$ and $\mathcal{F}_{0}$ is a Hilbert space with inner product $\mathcal{E}$. Suppose $v \in \mathcal{F}_{0}$ is $\mathcal{E}$-orthogonal to the space $\hat{\mathcal{F}}_{0}\left(\subset \mathcal{F}_{0}\right)$. Then

$$
\mathcal{E}(\psi(s), v)=0 \quad \text { for all } \psi \in C^{1}(\mathbb{R}) \text { with } \psi(0)=0 .
$$

By what has been proved, the left-hand side equals

$$
c_{0} \int_{I} \psi^{\prime}(s(x)) v^{\prime}(x) d x=c_{0} \int_{I} \psi^{\prime}(x) \frac{d}{d x}(v(t(x))) d x .
$$

Since this vanishes for any $\psi \in C_{0}^{1}(I)$, we have that $\frac{d}{d x} v(t(x))$ is constant and hence $v(x)=C s(x)$ for some constant $C$. Letting $\psi(x)=x$ in the above, we get $c_{0}^{2} C \int_{I} \frac{1}{\rho(x)} d x=0$, yielding that $C=0$.

We have proved that $\mathcal{F}_{0}=\hat{\mathcal{F}}_{0}$. Since any $v \in \mathcal{F}$ can be expressed as

$$
v(x)=v(0)+w(x) \quad \text { for } w(x)=v(x)-v(0)\left(\in \mathcal{F}_{0}\right)
$$

and $\hat{\mathcal{F}}$ contains constant functions, $\hat{\mathcal{F}}$ must be identical with $\mathcal{F}$.

Corollary 3. Let $\mathcal{D}$ be a linear subspace of $\mathcal{F}$ such that $\mathcal{D}$ is dense in $C(\bar{I})$ and, for any $\epsilon>0$, there exists a real function $\phi_{\epsilon}$ on $\mathbb{R}$ such that

$$
\begin{aligned}
& \phi_{\epsilon}(t)=t \forall t \in[0,1],-\epsilon \leq \phi_{\epsilon}(t) \leq 1+\epsilon \forall t \in \mathbb{R}, \text { and } \\
& 0 \leq \phi_{\epsilon}(t)-\phi_{\epsilon}\left(t^{\prime}\right) \leq t-t^{\prime} \text { whenever } t<t^{\prime},
\end{aligned}
$$

and $\phi_{\epsilon}(\mathcal{D}) \subset \mathcal{D}$. Then $\mathcal{D}$ is $\mathcal{E}_{1}$-dense in $\mathcal{F}$, namely, $\mathcal{D}$ becomes a core of the Dirichlet space $(\mathcal{E}, \mathcal{F})$ on $L^{2}(\bar{I}, \rho d x)$.

Proof. Let $\hat{\mathcal{F}}$ be the closure of $\mathcal{D}$ in the Dirichlet space $(\mathcal{F}, \mathcal{E})$. Then $(\hat{\mathcal{F}}, \mathcal{E})$ is a regular Dirichlet space on $C(\bar{I})$ (Theorem 3.1.1 of [1]).

For each $k=1,2, \cdots, \infty$, the space $C^{k}(\bar{I})$ of the restrictions to $\bar{I}$ of all $k$-times continuously differentiable functions on $\mathbb{R}$ is an example satisfying the conditions of Corollary 1. But the denseness of $C^{k}(\bar{I})$ in $\mathcal{F}$ is readily seen by mollifying each element of $\mathcal{F}$. We can consider less simple examples of $\mathcal{D}$ of Corollary 1.

For instance, denote by $m$ the Lebesgue measure on $\bar{I}$, take a Cantor-like set $A \subset \bar{I}([2])$ such that

$$
0<m(A \cap J)<m(J) \text { for any interval } J \subset \bar{I}, \quad \frac{1}{2}<m(A)<1,
$$

and let

$$
p(x)=\frac{1}{\gamma} \int_{0}^{x}\left(I_{A}(\xi)-I_{A^{c}}(\xi)\right) m(d \xi), \quad x \in \bar{I},
$$


where $\gamma=2 m(A)-1 . p(x)$ is extended to a continuous function on $\mathbb{R}$ by setting $p(x)=x$ outside $\bar{I}$. For $0 \leq a<b \leq 1$, we then define a function $p_{a, b}(x)$ on $\bar{I}$ by

$$
p_{a, b}(x)=\left(a \vee\left[a+(b-a) p\left(\frac{x-a}{b-a}\right)\right]\right) \wedge b .
$$

Since the family $\mathcal{D}_{0}=\left\{p_{a, b} ; 0 \leq a<b \leq 1\right\}$ separates points of $\bar{I}$, the linear lattice $\mathcal{D}$ (the linear space stable under the operations $\vee, \wedge$ ) generated by $\mathcal{D}_{0}$ and constant functions satisfies the conditions of the Kakutani-Krein Theorem (Corollary 1 on p. 10, 4]). However each element of $\mathcal{D}_{0}$ is not strictly monotone on any subinterval of $\bar{I}$ and it is not straightforward to see that this space $\mathcal{D}$ is dense in $\mathcal{F}$.

The diffusion process $\mathbf{M}=\left(X_{t}, P_{x}\right)$ considered in the proof of Theorem 1 is called a distorted Brownian motion on $\bar{I}$ and it admits the following expression:

$$
X_{t}-X_{0}=B_{t}+N_{t}, \quad t \geq 0, \quad P_{x} \text {-a.s. }
$$

for all $x \in \bar{I}$, where $B_{t}$ is a one dimensional standard Brownian motion starting at the origin and $N_{t}$ is a continuous additive functional of zero energy ([1]). In the special case that $\rho=1$, the distorted Brownian motion is the reflecting Brownian motion on $\bar{I}$ and the above expression is reduced to the Skorohod equation.

Corollary 3 says that, while the distorted Brownian motion $\mathbf{M}$ on $\bar{I}$ is uniquely determined by the function $\rho$ through the expression (1) of the form $\mathcal{E}$ on a space $\mathcal{D}$ satisfying conditions of the corollary, $\mathbf{M}$ does not depend on any specific choice of such a space $\mathcal{D}$.

\section{REFERENCES}

[1] Fukushima, M., Oshima, Y., Takeda, M., Dirichlet forms and symmetric Markov processes, Walter de Gruyter, Berlin-New York, 1994. MR 96f:60126

[2] Rudin, W., Real and complex analysis, McGraw-Hill, 1986. MR 88k:00002

[3] Ying, J., Killing and subordination, Proc. Amer. Math. Soc. 124, No. 7(1996), pp. 2215-2222. MR 97a:31005

[4] Yoshida, K., Functional analysis, Springer-Verlag, 1965

Department of Mathematics, Kansai University, Osaka 564-8680, Japan

E-mail address: fuku@ipcku.kansai-u.ac.jp

Department of Mathematics, Fudan University, Shanghai 200433, People's Republic OF CHINA

E-mail address: jying@math.zju.edu.cn 